\title{
Theoretical and Experimental Investigation of the Dissipated and Stored Energy Ratio in Iron under Quasi-Static and Cyclic Loading
}

\author{
O. Plekhov, ${ }^{1, \mathrm{a} a}$ S. Uvarov, ${ }^{1, \mathrm{~b}}$ and O. Naimark ${ }^{1, \mathrm{c}}$ \\ ${ }^{1}$ Institute of Continuous Media Mechanics, RAS-Laboratory of Physical Foundations of Strength, \\ Russian Academy of Sciences, Perm, Russia \\ apoa@icmm.ru, b usv@icmm.ru, ${ }^{\mathrm{c}}$ naimark@icmm.ru
}

The problem of energy storing (cold work accumulation) in metals was intensively investigated both theoretically and experimentally during all last century but a general theoretical conception of the process was not created. This work is devoted to an experimental investigation of energy dissipation in metals under plastic deformation and to the development of a thermodynamic model to study the cold work accumulation under plastic deformation and failure. The proposed model is based on a statistical description of collective properties of mesoscopic defects and on dividing the plastic deformation into two parts (dissipative and structural). The structural plastic strain was considered as an independent thermodynamic variable that allowed us to determine the thermodynamic potential of the system. The derived constitutive relations were applied for numerical simulation of tensile and cyclic tests. The numerical results demonstrate a good agreement with experimental data.

Keywords: cold work, energy dissipation, mesodefect evolution.

Introduction. The kinetics of the microstructure of metallic materials has been the subject of much experimental investigations. The available data indicate that the deformation of metals, especially plastic flow, is characterized by high dislocation activity and specific mesodefect patterns. The evolution of these structures, accompanied by failure and rotation of mesovolumes of the material, leads to generation of high internal stresses and, as a consequence, to energy storage in a specimen. The problem of the dependence of the storage energy $W_{s t}$ on the plastic work $W_{p}=\widetilde{\sigma}:\left(\widetilde{\varepsilon}-\widetilde{\varepsilon}_{e}\right)$ is widely covered in literature [1-3] but their relation is still an open question. A generally accepted assumption, $W<0.2 W_{p}$, which is often justified by citing the early study of Taylor et al. [4], is hardly applicable to many mechanical processes [3].

The basic theoretical problem of the models describing the energy balance under plastic deformation is determination of new structure-sensitive parameters. The plastic deformation, conventionally considered to be such a parameter, cannot be interpreted as an independent thermodynamic variable.

Naimark et al. [5] developed an original method for describing the damage kinetics using a statistical description of a mesodefect ensemble. Based on the statistical description of the problem, it is possible to determine characteristic responses of solids with defects and to work out an appropriate constitutive model. We use this approach to define thermodynamic internal variables and to obtain nonlinear kinetic equations that describe the energy balance in metals under plastic deformation. The plastic deformation is divided into two parts ("pure" plastic deformation and "structural" or "potential" deformation), and only one part (the structural deformation) is interpreted as an independent thermodynamic variable. The obtained kinetic equations are used to describe the thermal behavior of metals (for example, of pure iron) subjected to tensile and cyclic tests. We present numerical simulation that incorporates our model and shows that theoretical predictions and experimental results are in good quantitative agreement.

Thermodynamic Model. A general thermodynamic process obeys the momentum balance equation and the first and second laws of thermodynamics. In the case of small deformations, these equations involve the following thermodynamic quantities: strain and 
stress tensors $\widetilde{\varepsilon}$ and $\widetilde{\sigma}$, heat supply $r$, and specific Helmholtz free energy $F$. Assuming the following kinematic relation for the material under study $\widetilde{\varepsilon}=\widetilde{\varepsilon}^{e}+\widetilde{\varepsilon}^{p}+\widetilde{p}+\widetilde{\beta}\left(T-T^{\prime}\right)$, where $\widetilde{\varepsilon}^{e}$ is the elastic strain tensor, $\widetilde{\varepsilon}^{p}$ is the plastic strain tensor (related to the defect motion), $\widetilde{p}$ is the defect-induced strain tensor (structural part of the plastic deformation), $\widetilde{\beta}$ is the tensor of the thermal expansion coefficient, and $T^{\prime}$ is the reference temperature, we can write the following equation for the solid temperature evolution:

$$
c \dot{T}=Q^{e}+Q^{p}+r+\nabla T
$$

where $Q^{e}=T F_{\widetilde{\varepsilon}^{e} T}: \dot{\widetilde{\varepsilon}}^{e}$ is heating due to the thermoelastic effect, $Q^{p}=\widetilde{\sigma}: \dot{\widetilde{\varepsilon}}^{p}+$ $\left(\widetilde{\sigma}-F_{\tilde{p}}\right): \dot{\tilde{p}}+T F_{\tilde{p} T}: \dot{\tilde{p}}$ represents the inelastic contribution to the heating, $c$ is the specific heat capacity, and $F_{x}$ denotes the derivative of $F$ with respect to $x$.

Analysis of the inelastic contribution to the heating gives the following relation for the stored energy rate:

$$
W_{s t}=\frac{-T F_{\tilde{p} T}+F_{\tilde{p}}}{\widetilde{\sigma}:(\dot{\vec{\varepsilon}} p+\dot{\vec{p}})} .
$$

To solve Eq. (2), one should determine the structural plastic deformation $\tilde{p}$.

The structural parameters associated with typical mesoscopic defects (microcracks, microshears) were introduced in [5] as the derivatives of the dislocation density tensor. Those defects are described by symmetric tensors of the form $\tilde{s}=\vec{s} \vec{v}$ for microcracks and $\tilde{s}=1 / 2 s\left(\vec{v} \vec{l}+\vec{l} \vec{v}^{T}\right)$ for microshears. Here $\vec{v}$ is the unit vector normal to the base of a microcrack or the microshear slip plane, $\vec{l}$ is the unit vector in the direction of shear, and $s$ is the microcrack volume or the shear intensity for microshear. The average of the "microscopic" tensor $\tilde{s}$ gives the macroscopic tensor of the microcrack or the microshear density $\tilde{p}=n\langle\tilde{s}\rangle$, where $n$ is the defect concentration.

Statistical description of the microcrack (microshear) ensemble was developed in terms of the solution of the Fokker-Plank equation in the phase space of the possible states of the microscopic variable $\tilde{s}$ linking the size $s$ and the $\vec{v}, \vec{l}$ orientation modes. The obtained solution allowed the definition of the part of the free energy caused by defects $F_{\tilde{p}}$. The "equilibrium" correlation of the defect density tensor and the applied stress is given by the formula (for a one-dimensional case) $\partial F_{p}(\sigma, p) / \partial p=0$. The solution to the latter relationship depends on a new structural-scaling parameter $\delta$. This parameter indicates the scale distribution of the defect density tensor in a specific volume and plays the role of the second structural variable related to the multiscale nature of damage accumulation.

Finally, assuming linear relations between thermodynamic forces and fluxes, we can obtain the following constitutive equations:

$$
\begin{aligned}
& \dot{\widetilde{\varepsilon}}^{p}=L_{\varepsilon^{p}} F_{\widetilde{\varepsilon}^{e}}+L_{\varepsilon^{p} p}\left(F_{\tilde{\varepsilon}^{e}}-F_{\tilde{p}}\right), \\
& \dot{\widetilde{p}}=L_{\varepsilon^{p}}\left(F_{\widetilde{\varepsilon}^{e}}-F_{\tilde{p}}\right)+L_{\varepsilon^{p} p} F_{\varepsilon^{e}}, \\
& \dot{\delta}=L_{\delta} F_{\delta} .
\end{aligned}
$$

One-Dimensional Tensile Loading. The experimentally investigated material was annealed iron. During all tests, an infrared camera (CEDIP Jade III MWR) was used to record the temperature field evolution on the specimen surface. The main technical 
characteristics of the camera are as follows: spectral range $3-5 \mu \mathrm{m}$, maximum picture size $320 \times 240$ pixels, maximum framing rate $500 \mathrm{~Hz}, \mathrm{NETD}<25 \mathrm{mK}$ at $300 \mathrm{~K}$, and digital conversion 14 bits. To increase the surface emissivity properties, the specimen surface was painted black (mat paint) after polishing.

The experimentally obtained temperature field was numerically processed to determine the space-time evolution of the heat sources and the stored energy value. The typical results are presented in Fig 1.

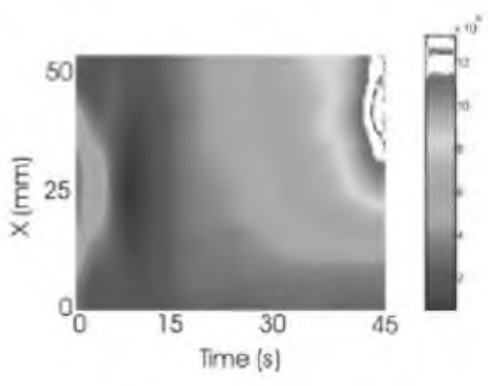

a

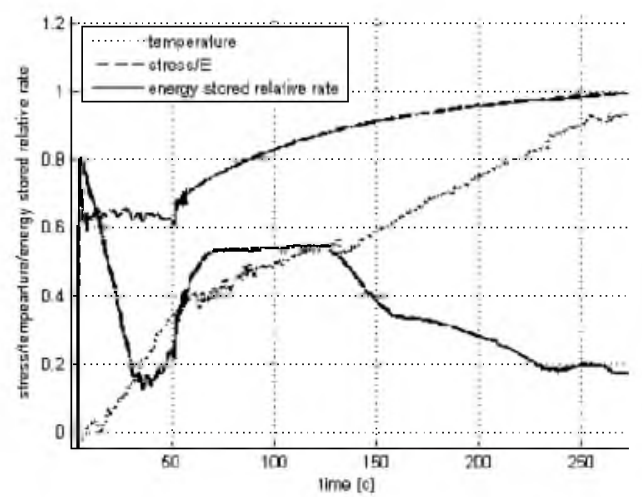

b

Fig. 1. Space-time evolution of the heat sources (in $\mathrm{W} / \mathrm{m}^{3}$ ) in the longitudinal specimen section (a), stress-strain, mean temperature variation, and stored energy rate curves (b) obtained during the test. (The stress and temperature difference were normalized by the corresponding maximum values. Maximum stress is $27 \mathrm{MPa}$, maximum temperature difference is $4^{\circ} \mathrm{C}$.)

To simulate the elastic-plastic transition accompanied by the heat wave propagation, the system of equations (1) and (3) together with the momentum balance equation was numerically solved. Figure 2 presents the experimentally and numerically determined space-time evolution of the heat sources in iron under elastic-plastic transition.

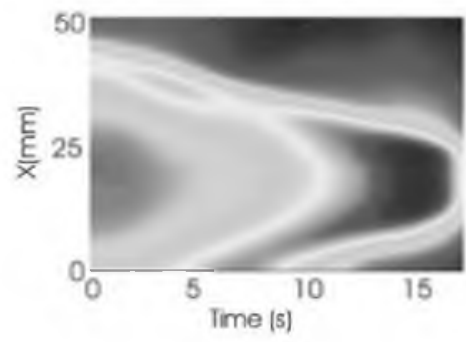

a

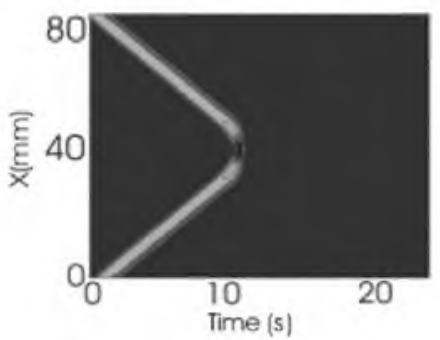

b

Fig. 2. Experimentally (a) and numerically (b) obtained space-time evolution of the heat sources in the longitudinal specimen section under elastic-plastic transition.

Cyclic Loading. The developed model allows us to simulate temperature evolution under cyclic loading. The main feature of cyclic loading is the creation of many different dislocation structures during the test. In terms of the model, it means that both structuresensitive parameters $\tilde{p}$ (deformation caused by defect initiation) and $\delta$ (describing the arrangement of dislocation ensemble) vary and interact during the test. Figure 3 presents the temperature evolution in iron during the numerical test. We obtain three (experimentally observed) stages of temperature evolution during the fatigue test: (I) initial temperature increase, (II) constant temperature region, (III) abrupt temperature increase before failure. 


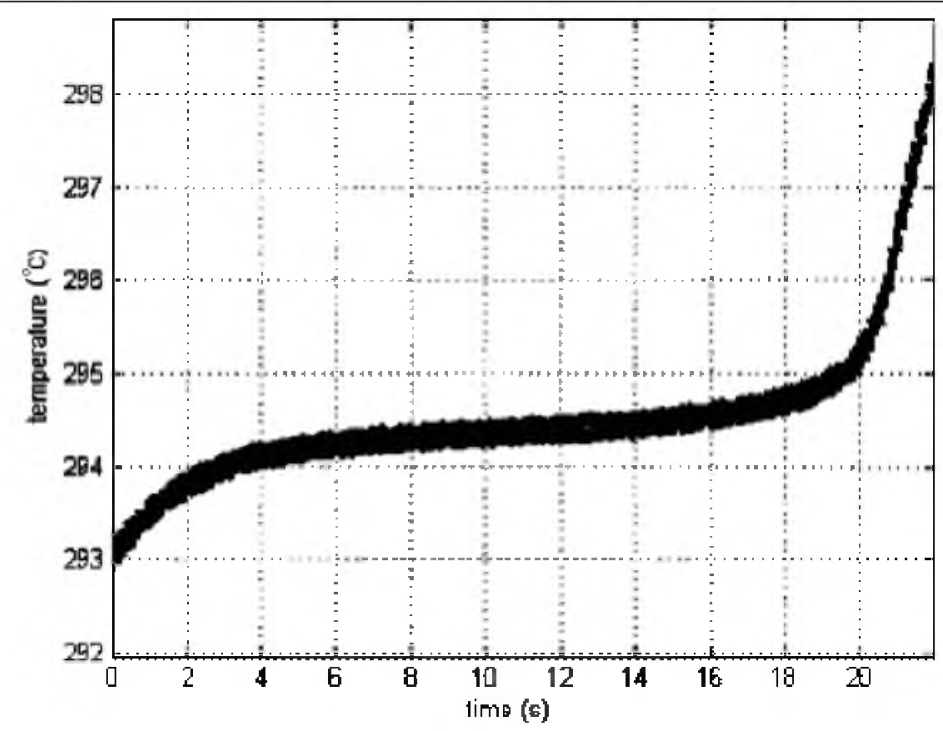

Fig. 3. Temperature evolution under cyclic loading during a numerical test.

Conclusions. Experimental and theoretical investigations of the energy storage and dissipation in metals allow us to propose a thermodynamic model to describe the energy balance under plastic deformation of metals. The key point of the model is the presentation of plastic deformation in terms of two variables: plastic strain tensor (related to the dissipation effect) $\widetilde{\varepsilon}^{p}$ and defect-induced strain tensor (related to the stored energy) $\widetilde{p}$. This makes it possible to consider the structure-related part of plastic deformation as a thermodynamic variable and to formulate a corresponding nonequilibrium thermodynamic potential (free energy). The developed approach has been successfully applied to the simulation of nonlinear thermal effects observed under quasi-static and cyclic loading of iron.

Acknowledgments. The authors thank the laboratory LAMEFIP ENSAM (personally Dr. T. PalenLuc and Dr. N. Saintier) for the help in the experimental investigations. The work was partly supported by the grants of RFBR (05-08-33652, 07-08-96001, 07-05-96019).

1. M. B. Bever, D. L. Hilt, and A. L. Titchener, Prog. Mater. Sci., 17, 1 (1973).

2. R. Kapoor and S. Nemat-Nasser, Mech. Mater., 27, 1 (1998).

3. P. Rosakis, A. J. Rosakis, G. Ravichandran, and J. Hodowany, J. Mech. Phys. Solids, 48, 581 (2000).

4. W. S. Farren and G. I. Taylor, Proc. Roy. Soc. London, A107, 422 (1925).

5. O. Naimark, M. Davydova, O. Plekhov, and S. Uvarov, Phys. Mesomech., 2, 43 (1999).

Received 28. 06. 2007 